\section{Keratoacanthoma centrifugum marginatum: unresponsive to oral retinoid and successfully treated with wide local excision}

\author{
Kapildev Das,' Nilay Kanti Das,' \\ Vikram Singh Rathore, ${ }^{2}$ Sourav Kundu, ${ }^{3}$ \\ Sourav Choudhury, ${ }^{4}$ Ramesh Chandra \\ Gharami,' Pijush Kanti Datta' \\ 'Department of Dermatology, Medical \\ College and Hospital, Kolkata; \\ 'Department of Plastic Surgery, Medical \\ College and Hospital, Kolkata; \\ ${ }^{3}$ Department of Dermatology, MGM \\ Medical College, Kishangang; \\ ${ }^{4}$ ESI Hospital, Manicktala, Kolkata, India
}

\section{Abstract}

We describe a case of a 65 -year-old male presenting with a large plaque with a rolled-out interrupted margin, atrophic center, and island of normal skin over the left arm. It grew peripherally with central healing, and there was a history of recurrence after inadequate excision. Investigations ruled out other clinical mimickers; namely, squamous cell carcinoma, lupus vulgaris, botryomycosis, and blastomycosis-like pyoderma. Histopathological sections showed irregularly shaped craters filled with keratin and epithelial pearl but no evidence of granuloma or cellular atypia. Clinicopathological correlation proved the lesion to be keratoacanthoma centrifugum marginatum (KCM), a rare variant of keratoacanthoma, which spreads centrifugally, attains a huge size, and never involutes spontaneously. Treatment of KCM has been a problem always and, in our case, systemic retinoid (acitretin for three months) proved ineffective. The patient also had a history of recurrence following surgical intervention previously, necessitating wide excision to achieve complete clearance of tumor cells. Hence, after failure of retinoid therapy, the decision of excision with a 1-centimeter margin was taken and the large defect was closed by a split thickness skin graft. The graft uptake was satisfactory, and the patient is being followed-up presently and shows no signs of recurrence after six months, highlighting wide local excision as a useful treatment option.

\section{Introduction}

Keratoacanthoma is a rapidly evolving cutaneous tumor composed of keratinizing squa- mous cells originating from the supra-seboglandular portion of the hair follicle. It is a disease of adult populations with males outnumbering females. ${ }^{1}$ Keratoacanthoma may be solitary or multiple but the most common form is the classical solitary variety, which occurs on the exposed body parts of the elderly person, and involutes by itself even if left untreated. Multiple keratoacanthomas, on the other hand, develop sporadically (e.g. eruptive keratoacanthomas of the Grzybowski type, or the familial syndromes like Muir-Torre syndrome, or its incomplete form, Ferguson-Smith syndrome). ${ }^{1}$ Keratoacanthoma centrifugum marginatum (KCM) is among the rare varieties and, in contrast to the classical variety, does not show any tendency of spontaneous regression. Lack of spontaneous regression is also a feature of another rare variant of keratoacanthoma, the giant keratoacanthoma, from which KCM is differentiated by the absence of downward vertical spread with destruction of the underlying tissue. Progressive peripheral extension with a raised rolled-out margin and atrophy at the center of the lesion is a characteristic feature of the KCM. ${ }^{1,2}$ The most common site of KCM is reported to be the dorsum of the hands and legs. $^{3}$

The etiology of KCM is multifactorial, which includes chronic ultraviolet ray exposure, the smoking habit, and contact with chemical carcinogens like pitch, mineral oil, tar, etc. It has been reported also in association with trauma and vaccination. ${ }^{1}$ The role of human papilloma virus (HPV) in keratoacanthoma remains inconclusive, with one study finding evidence of HPV infection by polymerase chain reaction, ${ }^{4}$ whereas another study failing to detect any virus material. ${ }^{5}$

\section{Case Report}

A 65-year-old healthy male, a cultivator by occupation, presented with a solitary painful plaque involving his left arm and forearm. It started spontaneously four years previously as a hyperpigmented nodule on the extensor aspect of the left forearm, which was operated on by a local doctor. The lesion recurred following the operation and gradually grew peripherally with healing of the center. The patient could not recall any history of trauma preceding the onset of the lesion, neither was there any family history of a similar disorder. Apart from tobacco smoking, there was no significant history of drug addiction, ingestion of halogenated compounds, or contact with chemicals.

On examination, a large single plaque $30 \times 10 \mathrm{~cm}$ in size was found occupying the left forearm (involving both dorsal and ventral aspects), the elbow, and the lower part of the
Correspondence: Nilay Kanti Das, Devitala Road, Majerpara, Ishapore, North 24 Paraganas, West Bengal, India, PIN- 743144.

E-mail: drdasnilay@gmail.com

Key words: keratoacanthoma centrifugum marginatum, retinoid, local excision.

Contributions: all authors contributed substantially to conception and design; acquisition, analysis and interpretation of data; drafting the article and revising it critically for important intellectual content; and final approval of the version to be published.

Conflict of interest: the authors report no conflicts of interest.

Received for publication: 14 July 2009.

Revision received: 15 December 2009.

Accepted for publication: 17 December 2009.

This work is licensed under a Creative Commons Attribution 3.0 License (by-nc 3.0).

(c) Copyright N.K. Das et al., 2010

Licensee PAGEPress, Italy

Dermatology Reports 2010; 2:e1

doi:10.4081/dr.2010.e1

left arm (Figure 1). The margin of the lesion was rolled out and interrupted, having hyperpigmented, firm, discrete, tender nodules and a cribriform pattern with comedonal orifices. The nodules had a central depression and foulsmelling cheesy material could be squeezed out on application of firm pressure on these nodules (Figure 2). The center of the plaque was atrophic and depigmented with intervening areas of normal skin. The lesion was not found to be adherent to the underlying structures and there was no regional lymphadenopathy or organomegaly.

Hematocrit, platelet, and leukocyte counts, and hepatic and renal function tests were within normal limits. A chest X-ray and ECG showed no abnormality. An X-ray of the left hand including wrist and elbow joints showed no bony involvement. The culture from the cheesy material revealed the presence of a gram-negative organism belonging to Proteus species. A biopsy taken from the margin of the lesion showed large irregularly shaped craters filled with keratin (Figure 3) and, from the base of the crater, irregular epidermal proliferations extending downward but not extending below the level of the sweat glands (Figure 4). At the periphery of the epidermal proliferations there was evidence of a thin layer of basophilic cells with the base of the crater containing epithelial pearls (Figure 5) and showing rich keratinization imparting a glassy 


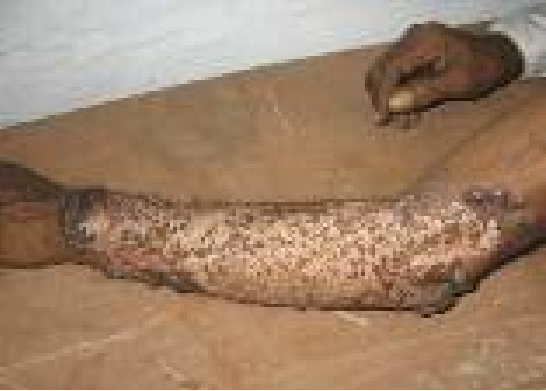

Figure 1. Plaque with rolled-out interrupted margin and atrophic center with island of normal skin located on the extensor aspect of the left forearm and lower part of the left arm.

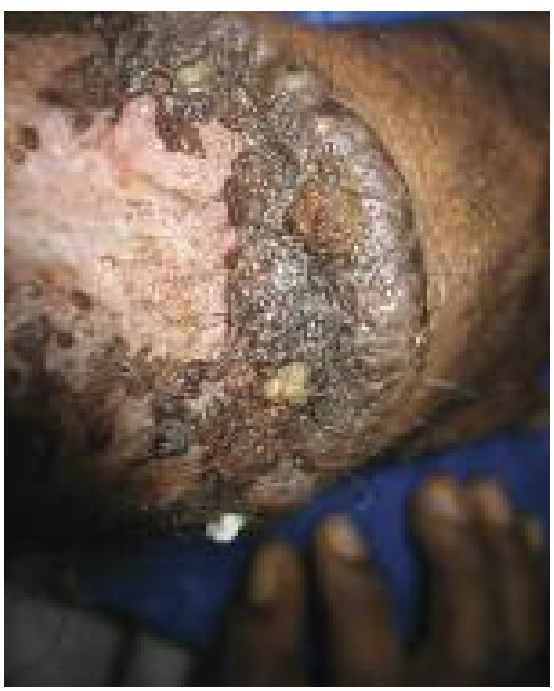

Figure 2. Close-up picture showing cheesy material exuding from (after being compressed laterally) a punctum overlying the margin of the lesion.

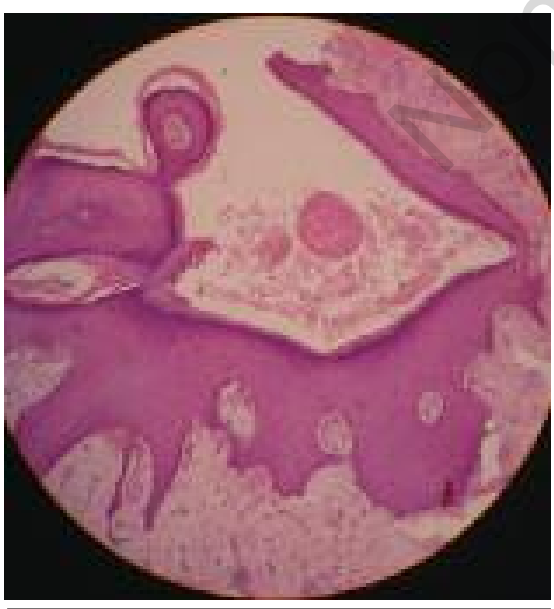

Figure 3. Histopathological section showing a large crater filled with keratin and having irregular epithelial proliferations extending into the papillary dermis. The basal layer is intact with no evidence of cellular atypia (hematoxylin and eosin stain; 100X magnification).

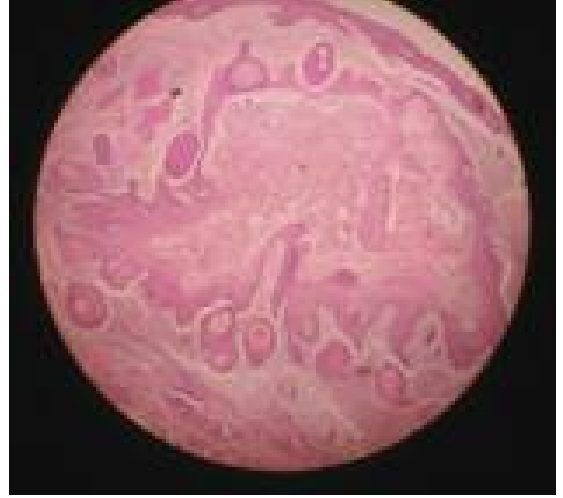

Figure 4. Cross-section of the crater showing a large cavity filled with keratin and irregular epidermal proliferations extending downward from its base, but not extending below the level of the sweat gland (hematoxylin and eosin stain; 40X magnification).

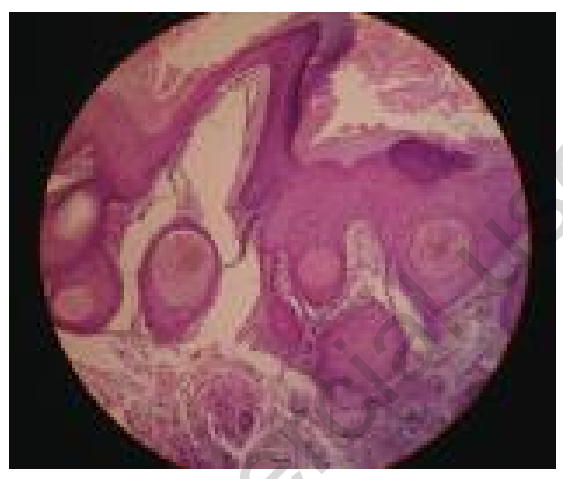

Figure 5. Histopathological section of the lateral wall of the epithelial proliferation showing a thin uninterrupted layer of basophilic cells and the base of the crater having epithelial pearls and well-keratinized cells giving a glassy appearance. No abnormal mitotic figures are detected and the papillary dermis shows scattered mononuclear cell infiltrates (hematoxylin and eosin stain; 100X magnification).

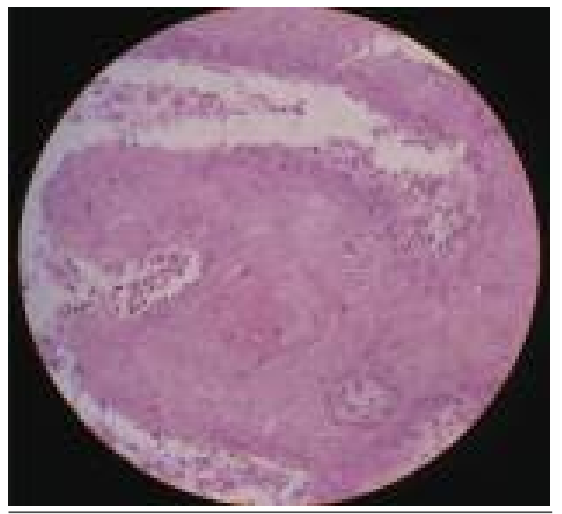

Figure 6. Higher magnification of the epidermal proliferation showing an eosinophilic, glassy appearing cell mass, marginated by a 1-2 layer of basophilic nonkeratinized cells (hematoxylin and eosin stain; 400X magnification).

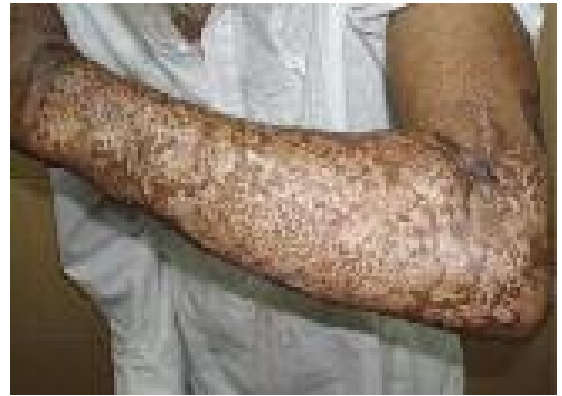

Figure 7. Six months after wide excision and repair of the nodules on the margins of the lesion showing the grafts (near the wrist and above the elbow) satisfactorily taken.

appearance (Figure 6). A mild to moderate degree of mononuclear cellular infiltrate was present within the dermis but mitotic figures were found rarely. There was no evidence of granuloma and no fungal element could be detected with PAS staining.

The clinicopathological correlation confirmed the case to be KCM and the patient was started on acitretin (initially $0.5 \mathrm{mg} / \mathrm{kg} /$ day then increasing the dose to $1 \mathrm{mg} / \mathrm{kg} /$ day after 15 days). Despite treatment for three consecutive months, the lesion showed no signs of improvement and a few new nodules appeared near the elbow crease. The failure necessitated that retinoid therapy be abandoned and operative intervention was planned. Plastic surgical reconstruction of the lesion was performed under regional block anesthesia. The nodules at the margin of the lesions were excised with a 1-cm margin, up to a depth of the antebrachial fascia. The defect was covered with a split thickness skin graft taken from the thigh. Postoperative graft uptake was satisfactory and the patient has been followedup for the past six months, showing no signs of recurrence (Figure 7).

\section{Discussion}

$\mathrm{KCM}$ is a very rare type of keratoacanthoma, and a PubMed search produced 31 entries of KCM in the world literature since its first description in $1962 .{ }^{6}$ Diagnosis of the condition was a real challenge in our case and meticulous clinical and histopathological examinations were required to differentiate it from several other conditions that closely mimic KCM. The clinical differential diagnoses include squamous cell carcinoma (SCC), lupus vulgaris (LV), botryomycosis, blastomycosis-like pyoderma (BLP), and pseu- 
doepitheliomatous hyperplasia (PEH). The fact that the lesion showed resolution in some places was a useful clinical clue in differentiating it from SCC. The presence of mitotic figures in our case cannot be taken as a marker of SCC as there were very few, although it has been reported that they may be associated with KCM. ${ }^{2}$ The large plaque with atrophy at the center of the lesion also prompted consideration of LV in the setting of the Indian subcontinent, but the absence of an underlying tubercular granuloma helped us to exclude this condition. The diagnostic dilemma was the finding of the presence of the Proteus organism within the lesions, which is a feature of botryomycosis. The absence of tumefaction or purulent discharge, with the histopathological finding failing to reveal any neutrophilic infiltration, grape-shaped granules, or SplendoreHoeppli phenomenon, ruled out the possibility of botryomycosis. ${ }^{7}$ Lastly, we had to exclude blastomycosis-like pyoderma (BLP). In fact, BPL may present also with a large verrucous plaque with pseudoepitheliomatous hyperplasia. ${ }^{8}$ However, we easily ruled out this condition as there were no pustules demonstrated clinically and no suppuration was found on histopathology; moreover, BPL usually affects immunosuppressed patients. ${ }^{9}$ Furthermore, PEH could be ruled out by the fact that it was not preceded by any other skin lesion, and by the histopathological finding of epidermal hyperplasia restricted to above the level of the sweat glands and the presence of a large crater, all of which are unlikely in the case of PHE. $^{2}$

After the exclusion of the clinical mimickers, KCM was diagnosed on the basis of classical clinical and histopathological findings. The appearance of recurrent nodular skin lesions with central clearing and progressive peripheral spreading on the distal extremities, ${ }^{6}$ in the setting of the histopathological findings of large irregularly shaped craters filled with keratin, with irregular epidermal proliferation having a glassy appearance and not extending below the level of the sweat glands, was diagnostic of KCM. The findings in our case were similar to those of previous case reports with the presence of large lesions and no tendency to spontaneous resolution. ${ }^{6}$ The margin of the lesion showing multiple comedonal orifices giving rise to a cibriform pattern, as seen in our case, may represent a unique phenomenon of KCM. We hypothesized that the typical appearance may arise as a result of sequential involvement of multiple adjacent hair follicles in a centrifugal fashion. It is an interesting finding and, if future case reports of this rare variant of keratoacanthoma document the same feature, then this hypothesis will be validated.

However, making the diagnosis was not the end and therapy was also a challenge. There are reports that KCM has been treated successfully with oral retinoids; ${ }^{10}$ hence, we started oral acitretin. After three months of high-dose therapy, it had to be abandoned because of no therapeutic improvement. Failure of retinoid as in our case has been documented earlier. ${ }^{11}$ The decision was taken in favor of surgical intervention and wide local excision was planned, considering the fact that the patient showed recurrence following previous inadequate excision. Following our intervention, the patient is showing no recurrence, highlighting that surgical intervention can be adopted as the preferred mode of therapy for KCM, keeping in mind that a wide excision is performed to prevent subsequent recurrence.

\section{References}

1. Cerroni L, Curl H. Keratoacanthoma. In: Fitzpatrick's Dermatology in General Medicine. 6th edn. Freedberg IR, EisenAZ, Wolff K, Austen FK, Goldsmith LA, KatzSA, editors. New York: McGraw Hill, 2003, pp.760-7.

2. Kirkham N. Tumors and cysts of the epidermis. In: Lever's Histopathology of the Skin. 9th edn. Elder DE, Elenitsas R, Johnson BL, Murphy GF, eds. Philadelphia: Lippincott Raven, 2005, pp.849-51.

3. Epidermal Nevi, Neoplasms and Cysts. In: Andrews' Disease of the Skin Clinical Dermatology. 10th edn. James WD, Berger TG, Elison DM, editors. Canada: Saunders Elsevier, 2006, pp.633-84.

4. His ED, Svoboda-Newman SM, Stern RA, et al. Detection of human papilloma virus DNA in keratoacanthoma by polymerase chain reaction. Am J Dermatopathol 1997; 19:10-5.

5. Lu S, Syrjanen SL, Huva VK, et al. Known HPV types have no association with keratoacanthomas. Arch Dermatol Res 1996; 288:129-32.

6. V'Ickova-Laskoska MT, Laskoski DS. Keratoacanthoma centrifugum marginatum: a rare atypical variant of keratoacanthoma. Clin Exp Dermatol 2008;33:259-61.

7. Lucas S. Bacterial Disease. In: Lever's histopathology of the skin. 9th edn. Elder DE, Elenitsas R, Johnson BL, Murphy GF, editors. Philadelphia: Lippincott Raven, 2005, pp.551-86.

8. Su WP, Duncan SC, Perry HO. Blastomycosis-like pyoderma. Arch Dermatol 1979;115:170-3.

9. Sawalka SS, Phiske MM, Jerajani HR. Blastomycosis-like pyoderma. Ind J Dermatol Venereol Leprol 2007;73:117-9.

10. Ogasawara Y, Kinoshita E, Ishida T, et al. A case of multiple keratoacanthoma centrifugum marginatum: response to oral etretinate. J Am Acad Dermatol 2003;48: 282-5.

11. Mangas C, Bielsa I, Ribera M, et al. A case of multiple keratoacanthoma centrifugum marginatum. Dermatol Surg 2004;30:8036 . 